\title{
Hypothermia therapy as an adjuvant therapy for severe ARDS.
}

\author{
Lee EK* \\ Intensive Care Medicine, Ng Teng Fong General Hospital, Singapore
}

\begin{abstract}
The management of severe ARDS (Adult respiratory distress syndrome) when maximal ventilator support fails has always been a challenge in places whereby Extracorporeal membrane oxygenation (ECMO) service may not be easily available or it may not be an option. Hypothermia therapy as a main mode of therapy for ARDS had been explored before but the result so far has not been convincing though Villar, had reported some success with hypothermic therapy for patients in 1993 but so far there have been a few recent trials that suggest otherwise. There are however a few case reports that had showed improvement in patients with severe ARDS refractory to other modes of conventional therapies. We would like to share the reports of 2 young males with severe ARDS from community and hospital acquired pneumonia with $\mathrm{PaO}_{2} /$ $\mathrm{FiO}_{2}$ ratio below 100 that was on maximal $\mathrm{AC} / \mathrm{VC}$ ventilator support of $\mathrm{FiO}_{2}$ of $100 \%$ with sedation and muscle relaxants. These 2 patients received adjuvant hypothermia therapy for 24 hours before they were gradually rewarmed to $37^{\circ} \mathrm{C}$ and weaned off ventilator support over the next 2 weeks. They recovered fully from their pneumonia and were subsequently discharged from the hospital.
\end{abstract}

Keywords: Hypothermia therapy, Respiratory, Patients, Mortality, Hyperinflammatory, Hypoinflammatory, Subphenotypes.

Accepted on March 19, 2019

\section{Introduction}

Acute respiratory distress syndrome (ARDS) is still a common and frequent fatal cause of respiratory failure among patients who are critically ill with an incidence of nearly 200000 cases per year in the USA alone, an estimated prevalence of $10 \%$ among critically ill patients worldwide, and a mortality of $30-40 \%$.

The definition of ARDS has been debated over time since the initial consensus in 1988 and currently it is based on the Berlin definition established in 2011 which is based on the degree of hypoxaemia, radiographic severity, respiratory system compliance, positive end-expiratory pressure and expired volume per minute.

Hypothermic therapy has been used as early as $450 \mathrm{BC}$ by the Greek physician, Hippocrates for soldiers with bleeding. He noticed that the soldiers had less bleeding when the wounds were covered with snow and that they had less mortality when they were kept cold as compared to those that were kept warm. Similarly the French chief medical officer of the French army, Baron Larrey, in 1814 during the Napoleon era discovered that the senior officers with wounds had higher mortality when they were kept warm versus the lower rank soldiers with similar wounds who were kept in the cold.

Other experimental therapy includes Professor William Osler who also had tried treating patients with typhoid fever in tubs of cold water. The fundamental principles behind hypothermic therapy are with the intentions to reduce cellular metabolism hence reduction of oxygen consumption and carbon dioxide production. It has also been shown that hypothermia will also affect the production of cytokines which will slow down the process of inflammation and this may help especially when the clinical condition is worsened by excessive inflammatory response. There is however no clinical evidence currently to support the use of hypothermic therapy for patients with sepsis or SIRS phenomenon.

An early study on ARDS using hypothermia therapy in 1993 by Villar and Slutsky [1] did show a significant reduction in mortality but the sample size unfortunately was very small. The relationship between body temperature alterations and critically ill patients is not well understood [2]. There are a few studies done recently that showed worsening outcomes with regards to the use of hypothermic therapy [2,3] in patients with sepsis and ARDS. However, this lack of evidence has not stopped people exploring hypothermic therapy in refractory ARDS [3]. This has also encouraged a recent large scale Root canal treatment (RCT) on hypothermic study in ARDS patients receiving muscle relaxants with funding from the University of Maryland with the finding yet to be published. Hypothermia has also been used in patients with ARDS using low tidal volume with the intention to minimize the rise of hypercapnia [4].

Carolyn et al. [5] in HARP-2 highlighted 2 distinct and consistent subphenotypes of ARDS and they are the hyperinflammatory and hypoinflammatory groups. These different subphenotypes responded differently to different treatments and these findings suggest that improved understanding of these subphenotypes could be crucial to future success in ARDS clinical trials [5,6].

The first patient is 28 year old who presented to the hospital after 8 days of coughing and fever as high as $39^{\circ} \mathrm{C}$. He was hypoxic on arrival in the Emergency department (ED) with the 
saturation below $90 \%$ on $100 \%$ non- rebreathing mask. He was tachypnic with rate above $40 / \mathrm{min}$ and a heart rate above $140 /$ min. His systolic blood pressure was barely above $80 \mathrm{mmHg}$ and diastole was slightly above $40 \mathrm{mmHg}$. His blood pressure did not improve despite 2 litres of crystalloid and a decision was made to intubate him immediately. His condition deteriorated, despite being on ventilator support with a $\mathrm{FiO}_{2}$ of 1.0 and $\mathrm{AC} /$ $\mathrm{VC}$ mode of ventilation with a peep of $12 \mathrm{~cm} \mathrm{H}_{2} \mathrm{O}$ (could not go any higher because of frequent hypotensive episodes). He was placed in a prone position to optimize his ventilation. Chest X-ray confirmed the presence of bilateral lower lobes pneumonia. His condition deteriorated further despite on maximal ventilator support and was unable to established ECMO transfer due to escalating infusion of vasoconstrictor. Hypothermic therapy was initiated 8 hours after he was intubated by the use of cold saline infusion, cold water through the nasogastric tube and cooling blanket. It took about 6 hours to bring his temperature to $35^{\circ} \mathrm{C}$ and about 8 hours to wean his $\mathrm{FIO}_{2}$ down to $60 \%$ after cooling was initiated. His heart rate also gradually decreased with improving systolic pressure and the down trending of vasoconstrictors (Figure 1).

He was placed in the supine position after 16 hours of prone position and he was cooled for a total of 24 hours at a range of $32-35^{\circ} \mathrm{C}$. However after 4 hours in the supine position with the same ventilator setting, he desaturated again and was placed in the prone position again. Fortunately his $\mathrm{FiO}_{2}$ requirement never went beyond $60 \%$. He became gradually better and on day 4 he was achieving a $\mathrm{P} / \mathrm{F}$ ratio above 250 . However he started to develop severe delirium and attempts to wean off the ventilator failed for the next 6 days. He was finally extubated on day $11^{\text {th }}$ in the ICU and weaned to room air over the next 2 days. There was a further delay in discharging him from the hospital as he had developed dysphagia and significant muscle weakness. He finally left for home after a total of 16 days in the hospital. There was no bacterial growth in his sputum or blood cultures. Influenza virus Type A came back positive from the sputum culture.

The second patient is a 24 year old male that had developed Guillian Barre syndrome after a viral infection. He was admitted to the hospital with bilateral lower limbs weakness. However, after 5 days in the hospital he developed hospital acquired pneumonia and ended with type 1 respiratory failure requiring intubation in the general ward. However, on arrival in the Intensive care unit (ICU) he was noted to have right sided pneumothorax and severe subcutaneous emphysema. His oxygenation deteriorated further despite on maximal ventilator support of $\mathrm{FiO}_{2}$ of $100 \%$ and $\mathrm{AC} / \mathrm{VC}$ mode of ventilation. $\mathrm{He}$ had a chest tube inserted into the right pleural space but despite this, his condition didn't improve. He was also hypotensive from the septic shock and was deemed too unstable to be transferred for ECMO or to be proned. Therapeutic hypothermic therapy was commenced after ECMO was declined by family due to financial constraints. The body temperature was lowered to $35^{\circ} \mathrm{C}$ from $39^{\circ} \mathrm{C}$ with a combination of cold saline and water through the nasogastric tube. He was also simultaneously cooled with a cooling blanket device. The pneumothorax persisted despite the insertion of a second chest tube and a diagnosis of bronchopleural fistula was established.

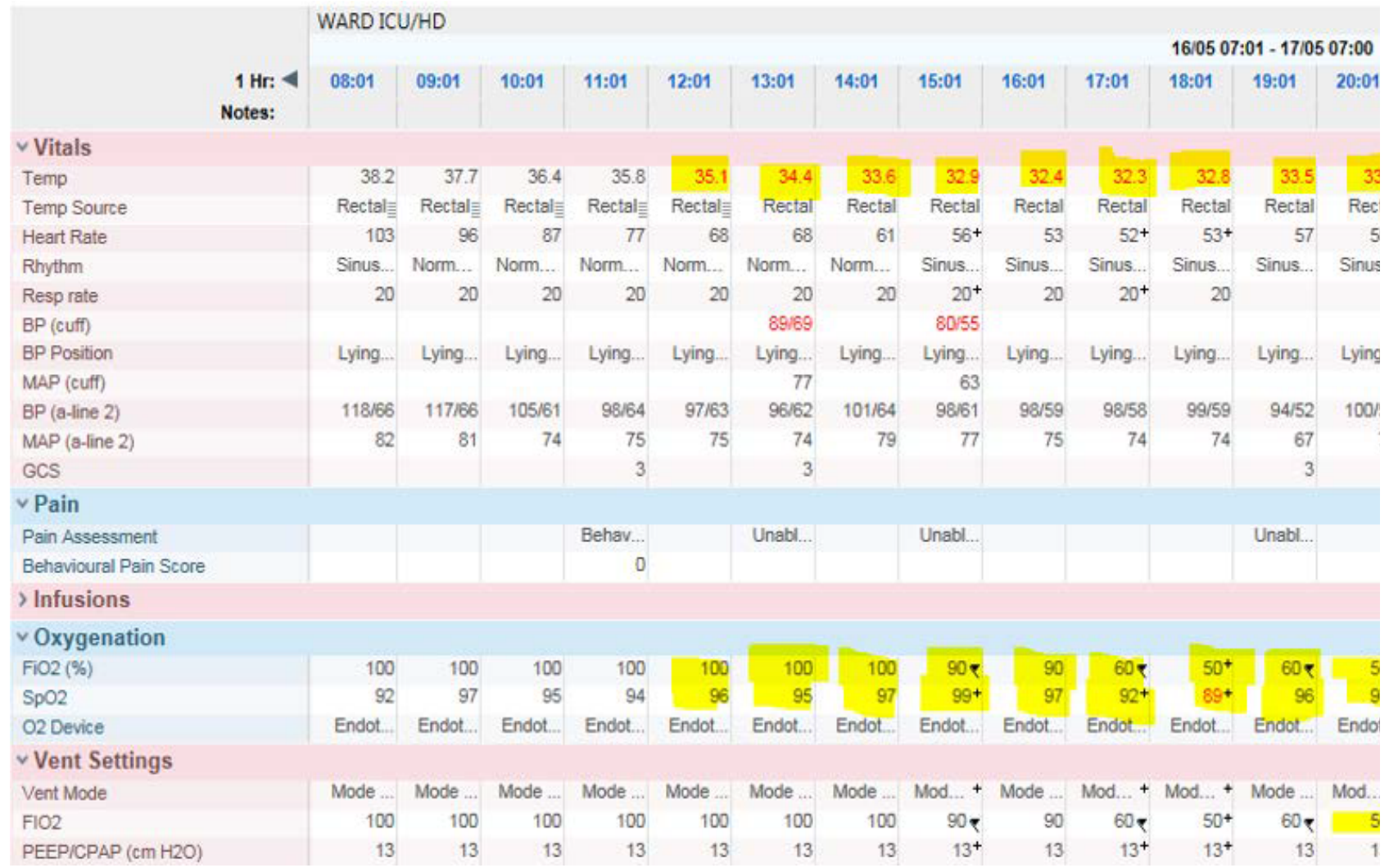

Figure 1. Parameters pertaining to first patient highlighted areas in the chart reflected the gradual improvement in the saturation and reduction in $\mathrm{FiO}_{2}$ as the patient's temperature dropped to $33.9^{\circ} \mathrm{C}$. 


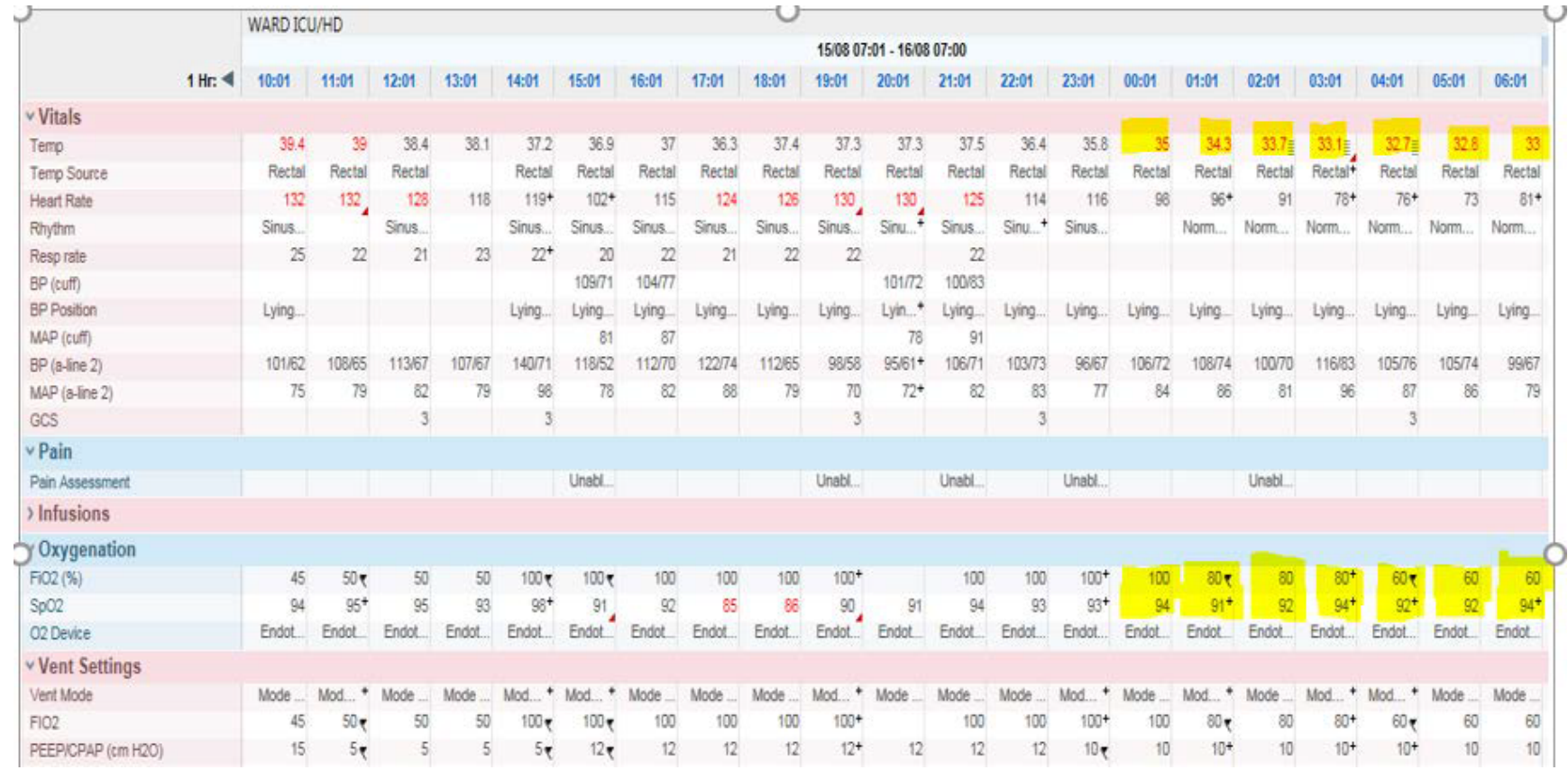

Figure 2. Parameters pertaining to second patient. Similarly the highlighted areas in the chart showed the concurrent improvement in saturation and decline in $\mathrm{FiO}_{2}$ as the temperature dropped $33^{\circ} \mathrm{C}$.

This patient was extremely refractory to hypothermic therapy and it took a total of 14 hours before his temperature came down to $35^{\circ} \mathrm{C}$ (Figure 2). There was also a concurrent improvement in the oxygenation and saturation. He was on an infusion of noradrenaline and vasopressin to maintain a Mean arterial pressure (MAP) of $65 \mathrm{mmHg}$. There was also a gradual improvement in the blood pressure and the vasoconstrictors were subsequently switched off after 8 hours of therapy. There was no evidence of any renal impairment throughout his ICU admission and care. This patient was rewarmed gradually and his temperature was brought back to $36^{\circ} \mathrm{C}$ after 24 hours of hypothermic therapy. The muscle relaxant, atracruim was switched off followed by the cessation of propofol and fentanyl infusion after his temperature was within the normal range. He was fully awake and established good tidal volume on minimum pressure support and Positive end-expiratory pressure (PEEP). He was subsequently extubated after 72 hours on the ventilator. Unlike the first patient, he had no delirium and complied with the physiotherapy and was on room air 24 hours after extubation. He was subsequently transferred to a cardiothoracic unit to manage the persistent broncho-pleural fistula in the right lung.

\section{Discussion}

We have done a literature review and there are more than 11 articles on hypothermic therapy in ARDS and many successful therapies were mentioned. However the latest study on hypothermic therapy in severe sepsis and respiratory failure that was published recently was stopped due to futility and increase in mortality.

There are at this stage no concrete evidence to support hypothermic therapy in severe sepsis and respiratory failure. Schell-Chaple et al. [2] highlighted in her study that there is a lack of standardization of measurement of temperature in most of these patients and inappropriate assignment of patients into the groups may affect the analysis and hence the conclusion that hypothermia has adverse outcomes. Fever as a biomarker is an indication of appropriate inflammatory response and whether the good outcome is attributed to the patient's underlying conditions or the fever itself is hard to differentiate. There is also no agreement on how long these patients should be kept on hypothermic therapy or the recommended ideal temperature in these articles that support the role of hypothermia.

The pathophysiology of ARDS can by characterized by various stages. The first phase begins with an injury to the alveolar capillary membrane which can occur as a direct injury such as pneumonia or indirectly as a result of extrapulmonary sepsis. It is followed by an exudative phase with a leak in capillary alveolar membrane causing a protein rich noncardiogenic pulmonary edema. Then there is an accumulation of leukocytes and macrophages with further release of cytokines in the pulmonary capillaries and airspaces. Concurrently there are injuries to the Type I alveolar cells with reduction of surfactant production and increased atelectasis. These can precipitate inflammatory vasoconstriction with worsening hypoxemia. The fibro-proliferative phase is initiated by the migration of fibroblasts which can initiate the repair of the damaged alveolar structure and re-establishment of the barrier. It is also associated with chronic inflammation and fibrosis of the alveoli membrane.

During sepsis there is an acute release of cytokines and neutrophils and macrophages which may precipitate ARDS and inhibits gaseous exchange. There is a possibility that a decline in the production of cytokines and immobilization of the macrophages and neutrophils may reduce the process of inflammation in the lung parenchyma and allow better gaseous exchange. This may contribute to better oxygen saturation. It will also at the same time allow the antibiotics to exhibit its bactericidal effects efficiently. The vasoconstrictive effect of hypothermia also helps to reduce the dosing of the vasoconstrictors. The bradycardic effects of hypothermia 
actually reduced the heartrates of these two patients and may have indirectly reduced oxygen consumption.

It is known that there is a $10 \%$ decrease in body metabolism per degree drop in temperature and this may account for the decrease in oxygen consumption. This may allow more oxygen to be delivered to the site of injury for better tissue repair.

Other side effect such as coagulopathy was not present in these 2 patients and there were no clinical evidence of bleeding from the central line sites or from the chest drains that were inserted. Renal function was also preserved in these patients and despite an initial acute kidney injury in both patients, it improved gradually as the vasoconstrictors were stopped and patients became haemodynamically stable.

We had tried to optimize the ventilator support for the above two patients to the best of our capability. The main therapy for ARDS in our department is still the trial of repeated titration of higher PEEP with a tidal volume of 6 to $8 \mathrm{ml} / \mathrm{kg}$ of tidal volume on ACVC mode. This is followed by either prone position ventilation or ECMO. The ECMO service was not an option for them due to financial constraints. We have not established any protocol for hypothermia in ARDS and these are the first 2 cases that we had managed with hypothermia. We have extrapolated our experience on therapeutic temperature management of post cardiac arrest patients and applied on these two patients with references to others trials done.

Gagandeep et al. [4] also wrote a similar report of a young man with ARDS as a result of lung contusion and fracture limbs that could not be fully proned or placed on ECMO recovered well after he was cooled to $35^{\circ} \mathrm{C}$ for 12 hours.

There are at this stage many conflicting views with regard to hypothermic therapy for ARDS. There is no strong evidence to support its usage as a sole therapy and it should only be considered as an adjuvant therapy in refractory ARDS as stated in some of the case reports.
There may be a need to identify the 2 distinct subphenotypes in subsequent clinical trials of hypothermia therapy on ARDS as Carolyn et al. [5] in HARP-26 highlighted that subphenotypes may respond differently to the same therapy.

\section{Conclusion}

We are in the progress of refining our therapy and we are limited by the number of ARDS cases we have. We hope that in the future with further improvement and information available we should be able to achieve better data with better titration of the temperature and duration of hypothermia therapy. These 2 case reports have highlighted that hypothermia therapy may have a role in refractory ARDS.

\section{References}

1. Villar J, Slutsky AS. Effects of Induced Hypothermia in Patients with Septic ARDS. Resuscitation. 1993;26(2):18392.

2. Schell-Chaple HM, Puntillo KA, Matthay MA, et al. Body Temperature and Mortality in Patients with ARDS. Am J Crit Care. 2015;24(1):15-23.

3. Itenov TS, Johansen ME, Bestle M, et al. Induced Hypothermia in Patients with Septic Shock and Respiratory Failure. Lancet Respir Med. 2018;6(3):183-92.

4. Gagandeep D, Gopal PB, Kamat AS, et al. Induced hypothermia for trauma-related ARDS. Indian J Crit Care Med. 2015;19(6):353-5.

5. Carolyn CS, Delucchi KL, Sinha P, et al. ARDS subphenotypes and Differential response to simvastatin: secondary analysis of a randomized controlled trial. Lancet Resp Med. 2018;6(9):691-8.

6. Thompson BT, Chambers RC, Liu KD. Acute Respiratory Distress Syndrome(ARDS). N Engl J Med. 2017;377(1):56272.

\section{*Correspondence to:}

Lee EK

Senior Consultant, Intensive Care Medicine

Ng Teng Fong General Hospital

Singapore

Tel: +6594842705

E-mail: leeek2001@yahoo.com.sg 